\title{
The MSA Certification Program for Biological Electron Microscopy Technologists
}

\author{
Leona Cohen-Gould ${ }^{1}$
}

1. Microscopy \& Image Analysis Core Facility, Weill Cornell Medical College, New York, NY, USA.

Since 1978, MSA (then, EMSA) has offered an opportunity for people working in the field of biological transmission electron microscopy to obtain certification as an EM technologist [1]. The motivation behind the development of the program was to establish baseline standards for technical proficiency as a means by which people could validate their expertise in the fundamental applications of sample preparation (EM-related laboratory methods, sample fixation, processing, embedding, sectioning and imaging/microscope use) so that perspective employers would know that if they hired a person who held that certification, that person would be able to join a lab and perform the necessary procedures at a professional level without the need of extensive training [2]. The acronym CEMT was approved by the MSA Council to be used by certified technologists as a professional identification (eg: Leona CohenGould, MS, CEMT) for as long as the certification is valid. Certification is valid for 10 years and renewal is a matter of paying the fee and verifying continued employment in the field. One does not have to be an MSA member to be certified, but the fee is substantially higher for non-members.

To obtain certification, an applicant must meet educational and/or occupational qualifications as outlined on the MSA website (microscopy.org/education/programs), submit letters of recommendation from 2 experienced electron microscopists in supervisory positions, and must pass both written and practical examinations. The written exam consists of 100 multiple choice questions, composed by members of the Certification Board, that address all aspects of standard, biological transmission electron microscopy. A grade of $80 \%$ or higher is required. Once a candidate passes the written exam, s/he must submit the materials for the practical exam. These include specimen blocks from 3 different nonpathological and non-human tissues from cell culture, animals, or plants, written preparation protocols, sections on slides and grids from those blocks as well as publication-quality annotated images captured from the submitted grids, along with figure legends. The exam is graded independently by two members of the Certification Board. If the two grades are disparate, a third member of the Board will also grade the exam. Again, a grade of $80 \%$ or better must be attained. The written exam must be proctored by someone approved by the Certification Board Chair and the candidate must sign a Pledge of Independent Workmanship that is submitted along with the practical exam materials. There are two exam cycles each year, Spring and Fall. Each cycle has 3 deadlines: application submission, written exam and practical exam. The deadlines, application form and guidelines brochure are on the MSA website.

In recent years, many employers in clinical labs, pharmaceutical companies and in academia have not only become aware of this certification, but will seek a certified technologist to fill a position in their institutions. In some hospitals, certification has become a requirement for promotion or even continued employment. A successful candidate receives an official document, signed by the Certification Board Chair and a "CEMT" lapel pin [3]. As we move further and further into an age of "kit" based and automated science there is still a need for people who can do "hands-on" science. A skilled electron microscopy technologist is an exemplar of just such science. 
References:

[1] J Petrali, Microscopy Today 18(1) (2010) 40.

[2] Kysar, L Cohen-Gould Microscopy Today 21(5) (2013) p. 46.

[3] P Kysar, E Calomeni, Micros. Microanal. 20 Suppl 3 (2014) p. 2148.

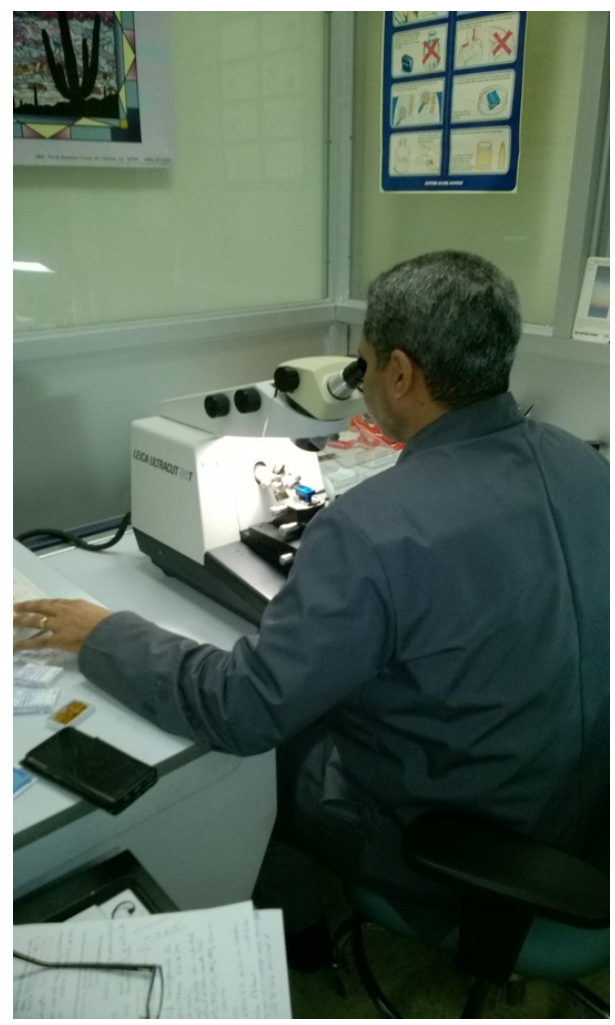

Figure 1. Working at the ultramicrotome.

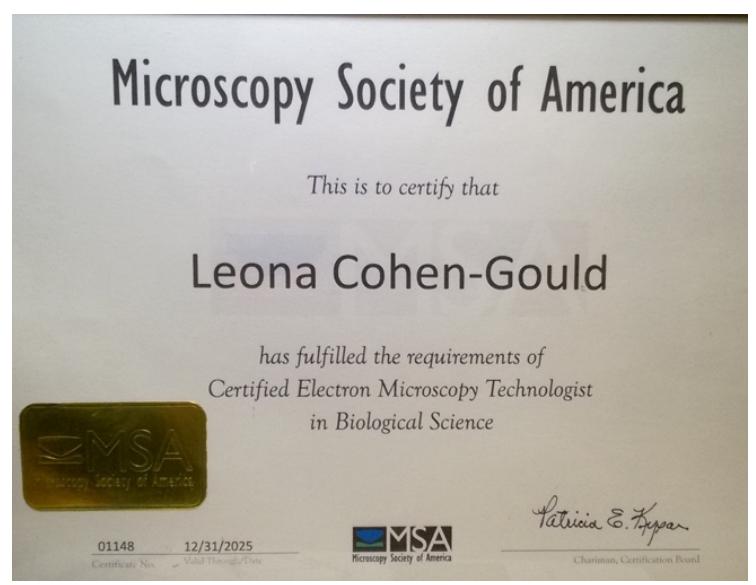

Figure 2. Official Certificate. 